\title{
ANGULAR DIFFERENTIAL CROSS-SECTION FOR ELECTRON TRANSFER IN ION-ION COLLISION
}

\author{
S. Bhattacharyya* , S.K. BhattacharyYa and S. Mitra \\ Gokhale Memorial Girls' College, Calcutta-700 020, India
}

(Received May 20, 1997; revised version October 15, 1997; in final form January 20,1998)

\begin{abstract}
Angular differential cross-sections for the formation of $\mathrm{He}^{0}$ in collision between fast $\mathrm{He}^{+}$ions are calculated using distorted wave Coulomb-Born approximation. The interaction potentials satisfy necessary Coulomb boundary conditions. In absence of any other theoretical results the present results are compared with the existing experimental data.
\end{abstract}

PACS numbers: $34.70 .+\mathrm{e}$

\section{Introduction}

Charge transfer in collision between positive ions is of theoretical and experimental interest being fundamental processes in astrophysical and laboratory plasmas, including Tokamak plasmas. Total cross-sections for charge exchange in one and two electron systems

$$
\mathrm{H}^{+}+\mathrm{He}^{+} \rightarrow \mathrm{H}^{0}+\mathrm{He}^{2+}
$$

and

$$
\mathrm{He}^{+}+\mathrm{He}^{+} \rightarrow \mathrm{He}^{0}+\mathrm{He}^{2+},
$$

respectively, have been investigated since many years, both theoretically [1-16] and experimentally [17-21]. Good agreement between theory and experiment was achieved. But the theoretical works on angular distribution of the scattered beams are scarce $[22,23]$ while first ever attempt on the experimental investigation on reaction (2) is by Giessen group [24] in Germany. The differential cross-section gives more sensitive probe by which one can have deep insight into the collision processes. Charge changing collision between $\mathrm{He}^{+}$ions in reaction (2) represents an unique ion-ion collision system which can be represented by hydrogen type wave function, the relative motion being distorted by Coulomb repulsion in the incident channel.

\footnotetext{
${ }^{*}$ Corresponding author's address: $370 / 1$ N.S.C. Bose Road, Calcutta-700 047, India.
} 
In this paper we formulate an expression for angular differential cross-section (ADCS) for the electron capture in the reaction (2). There has been as yet no theoretical work on this process. We use Coulomb-Borh approximation with the total interaction potentials as in the paper [23] for reaction (1). The post interaction includes two $(e-\alpha)$ attraction terms and one $(\alpha-\alpha)$ repulsive term. The prior form of the interaction contains interactions between $(\alpha-\alpha),(\alpha-e)$ and $(e-e) .(\alpha-\alpha)$ term is found to dominate both the channels. As such the post-prior discrepancy is small. The potentials are so chosen that the boundary conditions in the asymptotic limit are satisfied. This causes modification in the effective charge of the interacting systems. The Taylor series expansion of the potential terms provides convenient tools in writing the interaction potential terms in exponential forms. The exponential containing the momentum operator, provides translational factor associated with the relative motion.

Coulomb-Born approximation with distorted wave function is used in Ref. [23] to calculate the ADCS for the reaction (1). The results differed widely from those by Winter [22], but the post-prior discrepancy came out to be very small. However, there was no experiment on reaction (1) till then. In this paper we use the same methodology to calculate ADCS in the reaction (2) for which preliminary experimental results are available [24]. As in the experimental data [24], the maximum contribution for ADCS in (2) comes from a very small angular range $\left(0^{\circ}\right.$ to $\left.1^{\circ}\right)$ in the forward direction. ADCS decreases very rapidly with angle up to $0.2^{\circ}$, after which it becomes almost constant. We compute ADCS with the post and the prior interaction form of the potential. The difference in the ADCS for post and prior interactions exists in the forward direction which however is reduced appreciably as angle increases to $0.4^{\circ}$. This difference may be due to non-orthogonality between the initial and the final wave functions. In absence of any other theoretical result, the present results are compared with the preliminary experimental data by Giessen group (Fig. 2).

\section{Mathematical formalism}

The charge transfer reaction under consideration is $\mathrm{He}^{+}+\mathrm{He}^{+} \rightarrow \mathrm{He}^{0}+$ $\mathrm{He}^{++}$, where the projectile and the target are the helium ions $\left[\mathrm{He}^{+}(1 s)\right]$.

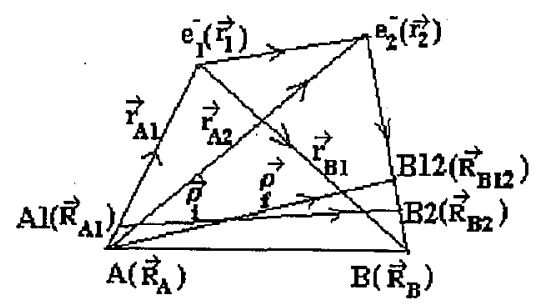

Fig. 1. Coordinate system: $A\left(\boldsymbol{R}_{A}\right)$ and $B\left(\boldsymbol{R}_{B}\right)$ are the nuclei of the projectile and target $\mathrm{He}^{+}(1 s)$ ions recpectively. $e_{1}^{-}\left(r_{1}\right)$ and $e_{2}^{-}\left(\boldsymbol{r}_{2}\right)$ are the two electrons bound respectively to $A$ and $B . A 1\left(\boldsymbol{R}_{A 1}\right)$ and $B 2\left(\boldsymbol{R}_{B 2}\right)$ are the $\mathrm{CM}$ of the $\left(A, e_{1}^{-}\right)$and $\left(B, e_{2}^{-}\right)$systems. $B 12\left(\boldsymbol{R}_{B 12}\right)$ is the $\mathrm{CM}$ of $\left(B, e_{1}^{-}, e_{2}^{-}\right)$system. 
In Fig. $1 \boldsymbol{R}_{A}, \boldsymbol{R}_{B}$ are the coordinates of the nuclei of the $\mathrm{He}^{+}$projectile $(A)$ and the target $(B)$ respectively. $\boldsymbol{r}_{1}$ and $\boldsymbol{r}_{2}$ are the coordinates of the two electrons $e_{1}^{-}$and $e_{2}^{-}$respectively. $\boldsymbol{R}_{A 1}$ and $\boldsymbol{R}_{B 2}$ are the coordinates of the centre of mass of $\left(\alpha_{A}-e_{1}^{-}\right)$system and $\left(\alpha_{B}-e_{2}^{-}\right)$system respectively, where $\alpha$ is the nucleus of the helium atom.

The total potential energy of the system is

$$
\begin{aligned}
U= & -\frac{Z_{A} e^{2}}{\left|\boldsymbol{r}_{1}-\boldsymbol{R}_{A}\right|}-\frac{Z_{A} e^{2}}{\left|\boldsymbol{r}_{2}-\boldsymbol{R}_{A}\right|}-\frac{Z_{B} e^{2}}{\left|\boldsymbol{R}_{B}-\boldsymbol{r}_{2}\right|}-\frac{Z_{B} e^{2}}{\left|\boldsymbol{R}_{B}-\boldsymbol{r}_{1}\right|} \\
& +\frac{Z_{A} Z_{B} e^{2}}{\left|\boldsymbol{R}_{B}-\boldsymbol{R}_{A}\right|}+\frac{e}{\left|r_{1}-r_{2}\right|} .
\end{aligned}
$$

The operator expressions for the kinetic energies of relative motions of the interacting systems in the initial and the final channels are respectively

$$
T_{\mathrm{i}}=-\frac{1}{2 \mu_{A 1}} \nabla_{A 1}^{2}-\frac{1}{2 \mu_{B 2}} \nabla_{B 2}^{2}-\frac{1}{2 \mu_{\mathrm{i}}} \nabla_{\mathrm{i}}^{2}-\frac{1}{2 M} \nabla_{R}^{2}
$$

and

$$
T_{\mathrm{f}}=-\frac{1}{2 \mu_{B 2}} \nabla_{B 2}^{2}-\frac{1}{2 \mu_{B 1}} \nabla_{B 1}^{2}-\frac{1}{2 \mu_{\mathrm{f}}} \nabla_{\mathrm{f}}^{2}-\frac{1}{2 M} \nabla_{R}^{2},
$$

where $\mu_{A 1}, \mu_{B 2}, \mu_{\mathrm{i}}, \mu_{\mathrm{f}}$ are the reduced masses associated with the relative coordinates $r_{A 1}, r_{B 2}, \rho_{\mathrm{i}}, \rho_{\mathrm{f}}$ respectively (Fig. 1), and $\nabla$ 's are with respect to the suffixed coordinates $\boldsymbol{R}_{A}, r_{A n}, r_{B n}(n=1,2), \rho_{\mathrm{i}}$ and $\boldsymbol{\rho}_{\mathrm{f}} . M$ and $R$ are respectively the mass and the CM coordinate of the interacting system.

$$
\begin{aligned}
& \boldsymbol{R}_{B 2}=\frac{M_{B} \boldsymbol{R}_{B}+m_{\mathrm{e}} \boldsymbol{r}_{2}}{M_{B}+m_{\mathrm{e}}}, \quad \rho_{\mathrm{i}}=\boldsymbol{R}_{B 2}-\boldsymbol{R}_{A 1}, \\
& r_{A 1}=r_{1}-\boldsymbol{R}_{A}, \quad \boldsymbol{r}_{A 2}=r_{2}-\boldsymbol{R}_{A} . \\
& \boldsymbol{R}_{A 1}=\frac{M_{A} \boldsymbol{R}_{A}+m_{\mathrm{e}} \boldsymbol{r}_{1}}{M_{A}+m_{\mathrm{e}}} \\
& M=M_{A}+M_{B}+m_{\mathrm{e}}+m_{\mathrm{e}}, \quad \boldsymbol{R}=\left[\boldsymbol{R}_{A 1}\left(M_{A}+m_{\mathrm{e}}\right)+\boldsymbol{R}_{B 2}\left(M_{B}+m_{\mathrm{e}}\right)\right] / M \\
& \boldsymbol{\rho}_{\mathrm{f}}=\boldsymbol{R}_{\boldsymbol{A}}-\boldsymbol{R}_{B 12}, \quad r_{B 1}=\boldsymbol{R}_{B}-\boldsymbol{r}_{1}, \quad \boldsymbol{r}_{B 2}=\boldsymbol{R}_{B}-\boldsymbol{r}_{2}, \\
& \boldsymbol{R}_{B 12}=\frac{M_{B} \boldsymbol{R}_{B}+m_{\mathrm{e}} \boldsymbol{r}_{1}+m_{\mathrm{e}} \boldsymbol{r}_{2}}{M_{B}+m_{\mathrm{e}}+m_{\mathrm{e}}} .
\end{aligned}
$$

The total Hamiltonian of the system in the initial channel is

$$
H=T_{\mathrm{i}}+U_{\mathrm{i}}=H_{0 \mathrm{i}}+V_{\mathrm{i}} \text {, }
$$

where $H_{0 \mathrm{i}}$ is the unperturbed part of the Hamiltonian and $V_{\mathrm{i}}$ is the perturbation part.

$$
\begin{aligned}
H_{0 \mathrm{i}} & =\left[-\nabla_{A 1}^{2} /\left(2 \mu_{A 1}\right)-\frac{Z_{A} e^{2}}{\left|r_{A 1}\right|}\right]+\left[-\nabla_{B 2}^{2} /\left(2 \mu_{B 2}\right)-\frac{Z_{B} e^{2}}{\left|r_{B 2}\right|}\right] \\
& +\left[-\nabla_{\mathrm{i}}^{2} /\left(2 \mu_{\mathrm{i}}\right)+\frac{\left(Z_{A}-1\right)\left(Z_{B}-1\right) e^{2}}{\left|\rho_{\mathrm{i}}\right|}\right]+\left(-\nabla_{R}^{2} / 2 M\right),
\end{aligned}
$$


where the differentials are with respect to the suffixed coordinates $r_{A 1}, r_{B 1}$ and $\rho_{\mathrm{i}}$.

$$
V_{\mathrm{i}}=-\frac{Z_{A} e^{2}}{\left|\boldsymbol{r}_{A 2}\right|}-\frac{Z_{B} e^{2}}{\left|\boldsymbol{r}_{B 1}\right|}+\frac{Z_{A} Z_{B} e^{2}}{\left|\boldsymbol{R}_{A B}\right|}+\frac{e^{2}}{\left|\boldsymbol{r}_{1}-r_{2}\right|}-\frac{\left(Z_{A}-1\right)\left(Z_{B}-1\right) e^{2}}{\left|\boldsymbol{\rho}_{\mathrm{i}}\right|} .
$$

Writing $r_{A 2}, r_{B 1}, \boldsymbol{R}_{A B}$ in terms of $\rho_{\mathrm{i}}, \mathrm{V}_{\mathrm{i}}$ can be written as

$$
\begin{aligned}
V_{\mathrm{i}}= & {\left[\frac{-Z_{A} e^{2}}{\left|\rho_{\mathrm{i}}+\left(m_{\mathrm{e}} / M_{A}\right)\left(\boldsymbol{r}_{B 2}-r_{B 1}\right)-r_{B 2}\right|}-\frac{-Z_{A} e^{2}}{\left|\rho_{\mathrm{i}}\right|}\right] } \\
& +\left[\frac{-Z_{B} e^{2}}{\left|\boldsymbol{\rho}_{\mathrm{i}}+\left(m_{\mathrm{e}} / M_{A}\right)\left(\boldsymbol{r}_{A 1}-\boldsymbol{r}_{A 2}\right)-r_{A 1}\right|}-\frac{-Z_{B} e^{2}}{\left|\boldsymbol{\rho}_{\mathrm{i}}\right|}\right] \\
& +\left[\frac{Z_{A} Z_{B} e^{2}}{\left|\boldsymbol{\rho}_{\mathrm{i}}+\left(m_{\mathrm{e}} / M_{A}\right)\left(\boldsymbol{r}_{A 1}-r_{A 2}\right)\right|}-\frac{Z_{A} Z_{B} e^{2}}{\left|\boldsymbol{\rho}_{\mathrm{i}}\right|}\right] \\
& +\left[\frac{e^{2}}{\left|\boldsymbol{\rho}_{\mathrm{i}}+\left(r_{B 1}-r_{A 2}\right)\right|}-\frac{e^{2}}{\left|\rho_{\mathrm{i}}\right|}\right] .
\end{aligned}
$$

Similarly, Hamiltonian in the final channel can be written as $H=T_{\mathrm{f}}+U=H_{0 \mathrm{f}}+V_{\mathrm{f}}$, where $H_{\text {of }}$ is the unperturbed part of the Hamiltonian and $V_{\mathrm{f}}$ is the perturbation part

$$
\begin{aligned}
H_{0 \mathrm{f}} & =\left[-\nabla_{B 2}^{2} /\left(2 \mu_{B 2}\right)-\frac{Z_{B} e^{2}}{\left|\boldsymbol{r}_{B 2}\right|}\right]+\left[-\nabla_{B 1}^{2} /\left(2 \mu_{B 1}\right)-\frac{Z_{B} e^{2}}{\left|\boldsymbol{r}_{B 1}\right|}\right] \\
& +\left[-\nabla_{\mathrm{f}}{ }^{2} /\left(2 \mu_{\mathrm{f}}\right)+\frac{Z_{A}\left(Z_{B}-2\right) e^{2}}{\left|\boldsymbol{\rho}_{\mathrm{f}}\right|}\right]+\left[-\nabla_{R}^{2} /(2 M)\right]+\frac{e^{2}}{\left|\boldsymbol{r}_{1}-r_{2}\right|}
\end{aligned}
$$

and

$$
V_{\mathrm{f}}=-\frac{Z_{A} e^{2}}{\left|r_{A 1}\right|}-\frac{Z_{A} e^{2}}{\left|r_{A 2}\right|}+\frac{Z_{A} Z_{B} e^{2}}{\left|\boldsymbol{R}_{A B}\right|}-\frac{Z_{A}\left(Z_{B}-2\right) e^{2}}{\left|\rho_{\mathrm{f}}\right|} .
$$

Writing $r_{A 1}, r_{A 2}, \boldsymbol{R}_{A B}$ in terms of $\rho_{\mathrm{f}}$ in $V_{\mathrm{f}}$,

$$
\begin{aligned}
V_{\mathrm{f}}= & {\left[\frac{-Z_{A} e^{2}}{\left|\rho_{\mathrm{f}}+\left(m_{\mathrm{e}} / M_{A}\right)\left(r_{B 2}-r_{B 1}\right)-r_{B 1}\right|}+\frac{Z_{A} e^{2}}{\left|\rho_{\mathrm{f}}\right|}\right] } \\
& +\left[\frac{-Z_{A} e^{2}}{\left|\rho_{\mathrm{f}}+\left(m_{\mathrm{e}} / M_{A}\right)\left(r_{B 2}-r_{B 1}\right)-r_{B 2}\right|}+\frac{Z_{A} e^{2}}{\left|\rho_{\mathrm{f}}\right|}\right] \\
& +\left[\frac{Z_{A} Z_{B} e^{2}}{\left|\rho_{\mathrm{f}}+\left(m_{\mathrm{e}} / M_{A}\right)\left(r_{A 1}-r_{A 2}\right)\right|}-\frac{Z_{A} Z_{B} e^{2}}{\left|\rho_{\mathrm{f}}\right|}\right] .
\end{aligned}
$$

As $\boldsymbol{\rho}_{\mathrm{i}}$ and $\boldsymbol{\rho}_{\mathrm{f}}$ tend to the asymptotic limit, each square bracket in (8a) and (10a) becomes identically zero. The interaction potentials $V_{\mathrm{i}}$ and $V_{\mathrm{f}}$ vanish in the asymptotic limit, and eventually satisfy boundary conditions.

Since $m_{\mathrm{e}} / M_{A} \ll 1$, we ignore the terms containing $m_{\mathrm{e}} / M_{A}$ as a factor in the interaction potentials $V_{\mathrm{i}}$ and $V_{\mathrm{f}}$ in (8a) and (10a). Using Taylor's expansions in (8a) and (10a) as in [23], and writing $\nabla_{\rho}$ in terms of momentum operator $p$,

$$
\nabla_{\rho}=\mathrm{i} p
$$


we get for $V_{\mathrm{i}}$ and $V_{\mathrm{f}}$

$$
V_{\mathrm{i}}=\frac{-Z_{A} e^{2}}{\left|\rho_{\mathrm{i}}\right|} \exp \left(-\mathrm{i} c \boldsymbol{P} \cdot \boldsymbol{r}_{B 2}\right)+\frac{-Z_{B} e^{2}}{\left|r_{B 1}\right|}+\frac{\left(Z_{A}+Z_{B}-1\right) e^{2}}{\left|\rho_{\mathrm{i}}\right|}+\frac{e^{2}}{\left|\boldsymbol{r}_{1}-r_{2}\right|}
$$

and

$$
V_{\mathrm{f}}=\frac{-Z_{A} e^{2}}{\left|\boldsymbol{\rho}_{\mathrm{f}}\right|} \exp \left(-\mathrm{i} c \boldsymbol{P} \cdot \boldsymbol{r}_{B 1}\right)+\frac{-Z_{A} e^{2}}{\left|\boldsymbol{\rho}_{\mathrm{f}}\right|} \exp \left(-\mathrm{i} c \boldsymbol{P} \cdot \boldsymbol{r}_{B 2}\right)+\frac{2 Z_{A} e^{2}}{\left|\boldsymbol{\rho}_{\mathrm{f}}\right|},
$$

where $c=1+m_{\mathrm{e}} / M_{A}$.

Eigenfunctions of the channel Hamiltonians $H_{0 \mathrm{i}}$ and $H_{\mathrm{Of}}$ are respectively,

$$
\psi_{\mathrm{i}}=\phi_{1}^{\mathrm{He}^{+}(1 s)}\left(r_{A 1}\right) \phi_{2}^{\mathrm{He}^{+}(1 s)}\left(r_{B 2}\right) \phi_{\mathrm{rel} .}^{\mathrm{i}}\left(K_{\mathrm{i}}, \rho_{\mathrm{i}}\right)
$$

and

$$
\psi_{\mathrm{f}}=\phi\left(r_{B 1}, r_{B 2}\right) \phi_{\text {rel. }}^{\mathrm{f}}\left(K_{\mathrm{f}}, \rho_{\mathrm{f}}\right),
$$

where $\phi_{1}^{\mathrm{He}^{+}(1 s)}\left(r_{A 1}\right), \phi_{2}^{\mathrm{He}^{+}(1 s)}\left(r_{B 2}\right)$, and $\phi\left(r_{B 1}, r_{B 2}\right)$ are the hydrogen type wave functions of the projectile and the target $\mathrm{He}^{+}(1 s)$ ions and $\mathrm{He}\left(1 s^{2}\right)$ atom, respectively. $\phi_{\text {rel. }}^{\mathrm{i}}\left(K_{\mathrm{i}}, \rho_{\mathrm{f}}\right)$ is the eigenfunction for relative motion of the colliding system in the initial channel, and in the final channel $\phi_{\text {rel. }}^{\mathrm{f}}\left(K_{\mathrm{f}}, \rho_{\mathrm{f}}\right)$ is a plane wave. Looking to the expressions (7), (9) of the unperturbed Hamiltonians $H_{0 \mathrm{i}}$ and $H_{0 \mathrm{f}}$ one finds that the effective nuclear charges for the relative motions are $\left(Z_{A}-1\right)$ and $\left(Z_{B}-1\right)$ in the incident channel and $Z_{A}$ and $\left(Z_{B}-2\right)$ in the final channel. Since for helium ion $Z_{A}=Z_{B}=2$, the wave function for relative motion $\phi_{\mathrm{rel}}^{\mathrm{i}}\left(\rho_{\mathrm{i}}\right)$ is Coulomb distorted while in the final channel the corresponding wave function $\phi_{\text {rel. }}^{\mathrm{f}}\left(\rho_{\mathbf{f}}\right)$ is undistorted. The Coulomb distorted wave function

$$
\begin{aligned}
\phi_{\text {rel. }}^{\mathrm{i}} & \left(K_{\mathrm{i}}, \rho_{\mathrm{i}}\right)=\left[1 /(2 \pi)^{3 / 2}\right] \exp (-\pi \eta / 2) \Gamma(1+\mathrm{i} \eta) \exp \left(-\mathrm{i} \boldsymbol{K}_{\mathrm{i}} \cdot \boldsymbol{\rho}_{\mathrm{i}}\right) \\
& \times_{1} F_{1}\left[-\mathrm{i} \eta, 1, \mathrm{i}\left(K_{\mathrm{i}} \rho_{\mathrm{i}}-\boldsymbol{K}_{\mathrm{i}} \cdot \boldsymbol{\rho}_{\mathrm{i}}\right)\right],
\end{aligned}
$$

where $\eta=\left(Z_{A}-1\right)\left(Z_{B}-1\right) \mu_{\mathrm{i}} /\left|\boldsymbol{K}_{\mathrm{i}}\right|$ and $\phi_{\mathrm{rel} .}^{\mathrm{f}}\left(\rho_{\mathrm{f}}\right)=\left[1 /(2 \pi)^{3 / 2}\right] \exp \left(-\mathrm{i} \boldsymbol{K}_{\mathrm{f}} \cdot \boldsymbol{\rho}_{\mathrm{f}}\right)$, where $\boldsymbol{K}_{\mathrm{i}}$ and $\boldsymbol{K}_{\mathrm{f}}$ are the relative momenta of the initial and the final channels, respectively. $\phi_{\text {rel. }}^{\mathrm{f}}\left(K_{\mathrm{f}}, \rho_{\mathrm{f}}\right)$ being a plane wave, the momentum operator $\boldsymbol{P}$ in (12) and (13) satisfies the equation

$$
\exp (-\mathrm{i} \boldsymbol{\lambda} \cdot \boldsymbol{P})\left|\phi_{\text {rel. }}^{\mathrm{f}}\left(K_{\mathrm{f}}, \rho_{\mathrm{f}}\right)\right\rangle=\exp \left(-\mathrm{i} \boldsymbol{\lambda} \cdot \boldsymbol{K}_{\mathrm{f}}\right)\left|\phi_{r e l .}^{f}\left(K_{\mathrm{f}}, \rho_{\mathrm{f}}\right)\right\rangle .
$$

The transition matrix element $V_{\mathrm{f}}^{\text {prior(post) }}$ of the prior (post) interaction $V_{\mathrm{i}}\left(V_{\mathrm{f}}\right)$ between the initial state $\psi_{\mathrm{i}}$ and the final state $\psi_{\mathrm{f}}$, is obtained in the Coulomb-Born approximation

$$
\begin{gathered}
V_{\mathrm{f}}^{\text {prior(post) }}=\int \psi_{\mathrm{f}} V_{\mathrm{i}, \mathrm{f}} \psi_{\mathrm{i}} \mathrm{d}^{3} r_{B 1} \mathrm{~d}^{3} r_{B 2} \mathrm{~d}^{3} \rho_{\mathrm{f}}=\int \phi\left(r_{B 1}, r_{B 2}\right) \phi_{\text {rel. }}^{\mathrm{f}}\left(K_{\mathrm{f}}, \rho_{\mathrm{f}}\right) V_{\mathrm{i}, \mathrm{f}} \\
\times \phi_{1}^{\mathrm{He}+(1 s)}\left(r_{A 1}\right) \phi_{2}^{\mathrm{He}+(1 s)}\left(r_{B 2}\right) \phi_{\text {rel. }}^{\mathrm{i}}\left(K_{\mathrm{i}}, \rho_{\mathrm{i}}\right) \mathrm{d}^{3} r_{B 1} \mathrm{~d}^{3} r_{B 2} \mathrm{~d}^{3} \rho_{\mathrm{f}} .
\end{gathered}
$$

We use both the prior and the post form of the interaction potentials to calculate the ADCS for charge transfer between $\mathrm{He}^{+}(1 s)$ and $\mathrm{He}^{+}(1 s)$ ions to produce $\mathrm{He}\left(1 s^{2}\right)$ atom in the ground state. The integrations involved are of the form 


$$
\begin{aligned}
V_{\mathrm{f}}(n) & =\int \phi\left(r_{B 1}, r_{B 2}\right) \phi_{\mathrm{rel} .}^{\mathrm{f}}\left(K_{\mathrm{f}}, \rho_{\mathrm{f}}\right)\left[\exp \left(-\mathrm{i} k_{\mathrm{f}} \cdot \lambda\right) /\left|\rho_{\mathrm{f}}\right|\right] \\
\times & \phi_{1}^{\mathrm{He}}{ }^{+(1 s)}\left(r_{A 1}\right) \phi_{2}^{\mathrm{He}+(1 s)}\left(r_{B 2}\right) \phi_{\mathrm{rel} .}^{\mathrm{i}}\left(K_{\mathrm{i}}, \rho_{\mathrm{i}}\right) \mathrm{d}^{3} r_{B 1} \mathrm{~d}^{3} r_{B 2} \mathrm{~d}^{3} \rho_{\mathrm{f}}
\end{aligned}
$$

where for

$$
\begin{array}{ll}
n=1, & \lambda=c r_{B 1}, \\
n=2, & \lambda=c r_{B 2}, \\
n=3, & \lambda=0,
\end{array}
$$

and

$$
\begin{aligned}
V_{\mathrm{f}}(4) & =\int \phi\left(r_{B 1}, r_{B 2}\right) \phi_{\text {rel. }}^{\mathrm{f}}\left(K_{\mathrm{f}}, \rho_{\mathrm{f}}\right)\left[1 /\left|r_{B 1}\right|\right] \phi_{1}^{\mathrm{He}^{+}(1 s)}\left(r_{A 1}\right) \phi_{2}^{\mathrm{He}^{+}(1 s)}\left(r_{B 2}\right) \\
& \times \phi_{\text {rel. }}^{\mathrm{i}}\left(K_{\mathrm{i}}, \rho_{\mathrm{i}}\right) \mathrm{d}^{3} r_{B 1} \mathrm{~d}^{3} r_{B 2} \mathrm{~d}^{3} \rho_{\mathrm{f}}, \\
V_{\mathrm{f}}(5) & \left.=\int \phi\left(r_{B 1}, r_{B 2}\right) \phi_{\text {rel. }}^{\mathrm{f}}\left(K_{\mathrm{f}}, \rho_{\mathrm{f}}\right)\left[1 / \mid r_{B 1}-r_{B 2}\right)\right] \phi_{1}^{\mathrm{He}^{+}(1 s)}\left(r_{A 1}\right) \phi_{2}^{\mathrm{He}^{+}(1 s)}\left(r_{B 2}\right) \\
& \times \phi_{\text {rel. }}^{\mathrm{i}}\left(K_{\mathrm{i}}, \rho_{\mathrm{i}}\right) \mathrm{d}^{3} r_{B 1} \mathrm{~d}^{3} r_{B 2} \mathrm{~d}^{3} \rho_{\mathrm{f}} .
\end{aligned}
$$

Using (19), (19a) and (19b) in (12) and (13) we obtain from (18) the interaction matrices for the prior and the post form of potential

$$
\begin{aligned}
& V_{\mathrm{fi}}^{\text {prior }}=\left(-Z_{A} e^{2}\right) V_{\mathrm{fi}}(2)+\left(-Z_{B} e^{2}\right) V_{\mathrm{fi}}(4)+\left(Z_{A}+Z_{B}-1\right) e^{2} V_{\mathrm{fi}}(3) \\
& \quad+e^{2} V_{\mathrm{fi}}(5), \\
& V_{\mathrm{fi}}^{\text {post }}=\left(-Z_{A} e^{2}\right) V_{\mathrm{fi}}(1)+\left(-Z_{A} e^{2}\right) V_{\mathrm{fi}}(2)+2 Z_{A} e^{2} V_{\mathrm{fi}}(3) .
\end{aligned}
$$

The integrations are shown in Appendix.

\section{Results and discussions}

The angular differential cross-section for charge transfer in the CM scattering angle is

$$
\mathrm{d} \sigma(\theta) / \mathrm{d} \Omega=\frac{\mu_{B 12} \mu_{A}}{(4 \pi)^{3}} 2 \pi \frac{\left|k_{\mathrm{f}}\right|}{\left|k_{\mathrm{i}}\right|}\left|V_{\mathrm{f}}\right|^{2} .
$$

We calculate the cross-section with both the post and the prior form of interaction potential. Usually, in charge transfer reaction, the energy and momentum transfer are very small. With no target recoil, in the projectile energy range under consideration, we find from (11A), $\left|\delta_{2}\right| /\left|\delta_{1}\right| \ll 1$, and approximate $\phi(\nu)=4 \pi / \delta_{1}$. The maximum contribution to the reaction comes from the small angular range in the forward direction. Correlations [25] are taken into consideration in the choice of the $\mathrm{He}\left(1 s^{2}\right)$ wave function in the final state. For collision energy $10 \mathrm{keV}(\mathrm{CM})$ the maximum contribution to ADCS comes from the range $0^{\circ}$ to $0.4^{\circ} \mathrm{CM}$-scattering angle. The results are compared with the preliminary data (Fig. 2) of the experiment [24] by Giessen group. 


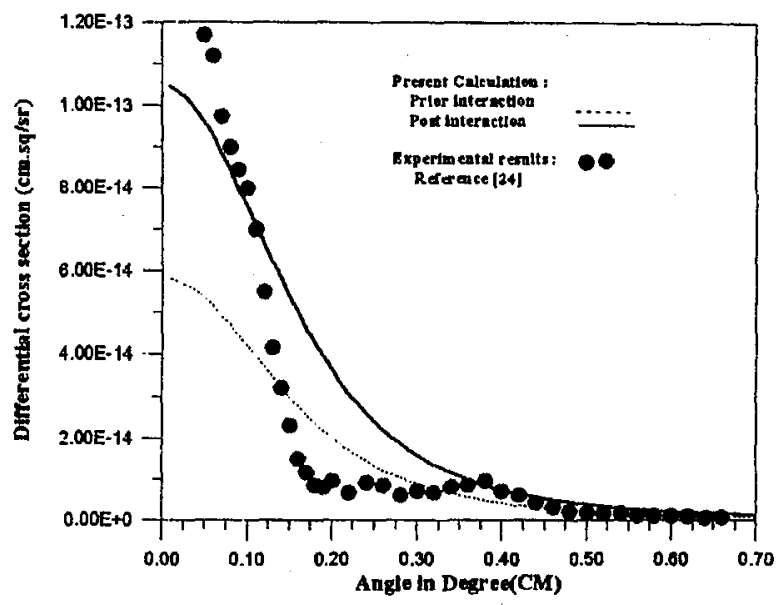

Fig. 2. The angular differential cross-section with respect to solid angle for charge transfer at $10 \mathrm{keV} \mathrm{CM}$ energy for the reaction $\mathrm{He}^{+}+\mathrm{He}^{+}=\mathrm{He}^{0}+\mathrm{He}^{++}$. Present calculation: prior interaction - - - , post interaction - - experimental results of Giessen group [24]

\section{Conclusion}

In this paper the Coulomb-Born approximation with Coulomb distorted wave function is used to calculate ADCS for the formation of $\mathrm{He}^{0}$ in collision between $\mathrm{He}^{+}$ions. A total interaction potential is considered. The post interaction potential contains $(\alpha-\alpha)$ and $(\alpha-e)$ interaction terms, in addition to which, the prior interaction potential contains $(e-e)$ term. Contribution from the $(\alpha-\alpha)$ term dominates the ADCS in both the channels. Though contribution from the $(e-e)$ term is much smaller than that from the $(\alpha-\alpha)$ term, yet the same is larger than that from $(\alpha-e)$ term. As such, ADCS in the post interaction channel is slightly greater than that in the prior interaction channel. However, beyond $0.5^{\circ}$, there is no post-prior discrepancy. The necessary boundary conditions, for asymptotic vanishing of the long range potentials, are achieved (8a), (10a).

In the experiment [24] as the absolute primary beam density profile in two dimensions could not be measured, the results were interpreted as preliminary and eventually some error in the measurement of ADCS is not unlikely. In principle, the present results for differential cross-section $\mathrm{d} \sigma / \mathrm{d} \Omega$ are plotted as a function of the CM-scattering angle at $10 \mathrm{keV}$. CM energy shows similar characteristic variation as obtained in the experimental results [24]. However, more such experiments are necessary to validate the present theoretical approach.

\section{Appendix}

The detailed calculations for $V_{\mathrm{f}}(n)$ are given below. The wave functions for $\mathrm{He}^{+}(1 s)$ ion in the initial state and that of $\mathrm{He}^{0}$ in the final state are respectively

$$
\phi_{1}^{\mathrm{He}^{+}(1 s)}\left(r_{A 1}\right)=N_{1} \mathrm{e}^{-\alpha_{1} r_{A 1}}, \quad \text { and } \quad r_{A 1}=\rho_{\mathrm{f}}-r_{B 1} \text {, }
$$


and

$$
\phi^{\mathrm{He}}\left(r_{B 1}, r_{B 2}\right)=N\left[\mathrm{e}^{-a r_{B 1}}+\mathrm{e}^{-b r_{B 2}}++\mathrm{e}^{-b r_{B 1}}+\mathrm{e}^{-a r_{B 2}}\right],
$$

where

$$
N_{1}=(1 / \sqrt{\pi})\left(Z_{A} / a_{0}\right)^{3 / 2}, \quad \alpha_{1}=Z_{A} / a_{0}
$$

and $N, a, b$ are obtained from Ref. [25].

$$
N^{2}=0.502571, \quad a=1.8850, \quad b=2.1832 \text {. }
$$

The wave function $\phi_{2}^{\mathrm{He}^{+}(1 s)}\left(r_{B 2}\right)$ of $\mathrm{He}^{+}$is equal to

$$
\phi_{2}^{\mathrm{He}+(1 s)}\left(r_{B 2}\right)=N_{2} \mathrm{e}^{-\alpha_{2} r_{B 2}} \text {, }
$$

where

$$
N_{2}=(1 / \sqrt{\pi})\left(Z_{B} / a_{0}\right)^{3 / 2}, \quad \alpha_{2}=Z_{B} / a_{0} .
$$

The integrals $V_{\mathrm{f}}(n)$ in (18), for $n=1$ to 3 , are shown below. Since $m_{\mathrm{e}} / M_{A} \ll 1$, from (6) one can approximate $\boldsymbol{R}_{A 1}=\boldsymbol{R}_{A}$ and $\boldsymbol{R}_{B 1}=\boldsymbol{R}_{B 12}=\boldsymbol{R}_{B}$, leading to the fact that $\rho_{\mathrm{i}}=\rho_{\mathrm{f}}=\rho$. On using atomic units

$$
\begin{aligned}
& V_{\mathrm{fi}}(n)=\int \phi\left(r_{B 1}, r_{B 2}\right) \phi_{\mathrm{rel} .}^{\mathrm{f}}\left(K_{\mathrm{i}}, \rho_{\mathrm{i}}\right)(1 /|\rho|) \exp \left(-\mathrm{i} \boldsymbol{c} \cdot \boldsymbol{K}_{\mathrm{f}}\right) \phi_{1}^{\mathrm{He}^{+}(1 s)}\left(\left|\boldsymbol{\rho}-r_{B 1}\right|\right) \\
& \quad \times \phi_{2}^{\mathrm{He}^{+}(1 s)}\left(r_{B 2}\right) \phi_{\mathrm{rel} .}^{\mathrm{i}}\left(K_{\mathrm{f}}, \rho_{\mathrm{f}}\right) \mathrm{d}^{3} r_{B 1} \mathrm{~d}^{3} r_{B 2} d^{3} \rho
\end{aligned}
$$

After integration over $\boldsymbol{r}_{B 1}$ and $\boldsymbol{r}_{B 2}$ we get

$$
V_{\mathrm{f}}(1)=T_{1}(a, b)+T_{1}(b, a),
$$

where

$$
\begin{aligned}
& T_{1}(a, b)=\iint N_{0}(2 \pi)^{-6} 8 \pi a 8 \pi b_{1}[|\boldsymbol{\rho}|]^{-1} J_{1}(K) \exp \left[\mathrm{i}\left(\boldsymbol{K}_{\mathrm{f}}-\boldsymbol{K}_{\mathrm{i}}\right) \boldsymbol{\rho}_{\mathrm{f}}\right] \\
& \quad \times_{1} F_{1}\left[-\mathrm{i} \eta, 1, \mathrm{i}\left(K_{\mathrm{i}} \rho-\boldsymbol{K}_{\mathrm{i}} \cdot \boldsymbol{\rho}\right)\right] \mathrm{d}^{4} \boldsymbol{\rho} \mathrm{d}^{3} \boldsymbol{K}, \\
& J_{1}(K)=\left[\left(a^{2}+L_{1}^{2}\right)^{-2}\left(b_{1}^{2}+L_{1}^{\prime 2}\right)^{-2}\left(K^{2}+\alpha_{1}\right)^{2}\right]^{-1} \exp (\mathrm{i} \boldsymbol{K} \cdot \boldsymbol{\rho}) \\
& =\left[A_{1}+f_{1}(K)\right]^{-2}\left(K^{2}+\alpha_{1}\right)^{-2} \exp (\mathrm{i} \boldsymbol{K} \cdot \boldsymbol{\rho}) \\
& =-\left(\mathrm{d} / \mathrm{d} A_{1}\right)\left\{\left[A_{1}+f_{1}(K)\right]^{-1}\left(K^{2}+\alpha_{1}\right)^{-2} \exp (\mathrm{i} \boldsymbol{K} \cdot \boldsymbol{\rho})\right\},
\end{aligned}
$$

where

$$
\begin{aligned}
& \boldsymbol{L}_{1}=\boldsymbol{K}_{\mathrm{f}}-\boldsymbol{K}, \quad \dot{L_{1}^{\prime}}=0, \\
& A_{1}=a^{2} b_{1}^{2}+b_{1}^{2} K_{\mathrm{f}}^{2}, \quad f_{1}(K)=b_{1}^{2} K^{2}-2 b_{1}^{2} K_{\mathrm{f}} K \cos \theta .
\end{aligned}
$$

Using Feynman identity

$$
1 /\left(a_{2}^{2} b_{2}\right)=2 \int x \mathrm{~d} x /\left[a_{2} x+b_{2}(1-x)\right]^{3}
$$


we get

where

$$
J_{1}(K)=-2\left(\mathrm{~d} / \mathrm{d} A_{1}\right) \int x \mathrm{~d} x \frac{\exp (\mathrm{i} \boldsymbol{K} \cdot \boldsymbol{\rho})}{\left\{\left[A_{1}+f_{1}(K)\right](1-x)+\left(K^{2}+\alpha_{1}\right) x\right\}^{3}},
$$

$$
\begin{aligned}
& N_{0}=N N_{1} N_{2}\left[1 /(2 \pi)^{3}\right]\left(-Z_{A} e^{2}\right) \exp (-\pi \eta / 2) \Gamma(1+\mathrm{i} \eta), \\
& b_{1}=b+1, \quad Z_{A}=2 \quad \text { and } \quad a_{0}=1 . \\
& \text { Using the integral formula } \\
& \int \exp (\mathrm{i} K \cdot \rho) \mu^{-3}\left(\nu^{2}+K^{2}\right)^{-3} \mathrm{~d}^{3} \boldsymbol{K}=-\mu^{-3}\left(\pi^{2} / 4 \nu\right)(\mathrm{d} / \mathrm{d} \nu)[\exp (-\nu r) / \nu],
\end{aligned}
$$

where from (8A) $\mu=x+b_{1}^{2}(1-x), \theta$ is the angle between $\boldsymbol{K}_{\mathrm{f}}$ and $\boldsymbol{K}_{\mathrm{i}}$, and

$$
\nu^{2}=\left[x+\left(a^{2} b_{1}^{2}+K_{\mathrm{f}}^{2} b_{1}^{2}\right)(1-x) / \mu\right]-\left\{\left[b_{1}^{2} K_{\mathbf{f}} \cos \theta(1-x)\right]^{2} / \mu^{2}\right\} .
$$

Hence from $(5 \mathrm{~A})$

$$
\begin{aligned}
& T_{1}(a, b)=N_{0} 32 a b_{1} \pi^{4}(2 \pi)^{-6}\left(\mathrm{~d} / \mathrm{d} A_{1}\right)\left[\int \mu^{-3} \nu^{-1}(\mathrm{~d} / \mathrm{d} \nu)(\phi(\nu) / \nu) x \mathrm{~d} x\right], \\
& \phi(\nu)=\int \exp \left[-\nu \rho+\mathrm{i}\left(\boldsymbol{K}_{\mathrm{f}}-\boldsymbol{K}_{\mathrm{i}}\right) \cdot \boldsymbol{\rho}\right][|\boldsymbol{\rho}|]^{-1} \\
& \quad \times_{1} F_{1}\left[-\mathrm{i} \eta, 1, \mathrm{i}\left(K_{\mathrm{i}} \rho-\boldsymbol{K}_{\mathrm{i}} \cdot \boldsymbol{\rho}\right)\right] \mathrm{d}^{3} \boldsymbol{\rho} .
\end{aligned}
$$

Using Nordsieck integrals [26] we get

$$
\begin{aligned}
& \phi(\nu)=\left(4 \pi / \delta_{1}\right)\left(1+\delta_{2} / \delta_{1}\right)^{i \eta} \\
& \delta_{1}=\nu^{2}+\left(\boldsymbol{K}_{\mathrm{f}}-\boldsymbol{K}_{\mathrm{i}}\right)^{2}, \quad \delta_{2}=2\left(\boldsymbol{K}_{\mathrm{f}} \cdot \boldsymbol{K}_{\mathrm{i}}-\boldsymbol{K}_{\mathrm{f}}^{2}-\mathrm{i} \nu K_{\mathrm{f}}\right),
\end{aligned}
$$

while

$$
\begin{aligned}
& \int \exp \left[-\nu \rho+\mathrm{i}\left(\boldsymbol{K}_{\mathrm{f}}-\boldsymbol{K}_{\mathrm{i}}\right) \cdot \boldsymbol{\rho}\right]{ }_{1} F_{1}\left[-\mathrm{i} \eta, 1, \mathrm{i}\left(K_{\mathrm{i}} \rho-\boldsymbol{K}_{\mathrm{i}} \cdot \boldsymbol{\rho}\right)\right] \mathrm{d}^{3} \boldsymbol{\rho} \\
& \quad=-(\mathrm{d} / \mathrm{d} \nu) \phi(\nu) .
\end{aligned}
$$

The integration over $x$ in $(9 \mathrm{~A})$ is carried out using trapezoidal rule with sufficiently small mesh $\Delta x$.

\section{Acknowledgment}

The authors are thankful to UGC, New Delhi for supporting the work through grant no. F10-100/90(RBB-II).

\section{References}

[1] R.E. Olson, J. Phys. B 11, L 227 (1978).

[2] C. Sinha, N.C. Sil, Phys. Lett. A 71, 201 (1979).

[3] D. Belkic, R. Gayet, A. Salin, Phys. Rep. 56, 279 (1979).

[4] T.G. Winter, G.J. Hatton, N.F. Lane, Phys. Rev. A 22, 930 (1980).

[5] W. Fritsch, C.D. Lin, J. Phys. B 15, 1255 (1982).

[6] T.G. Winter, Phys. Rev. A 25, 697 (1982). 
[7] J.F. Reading, A.L. Ford, R.L. Becker, J. Phys. B 15, 625 (1982).

[8] B.H. Bransden, C.J. Noble, J. Chandler, J. Phys. B 16, 4191 (1983).

[9] S. Datta, C.R. Mandal, S.C. Mukherjee, Can. J. Phys. 62, 307 (1984).

[10] S.L. Willis, G. Peach, M.R.C. McDowell, J. Banerjee, J. Phys. B 18, 3939 (1985).

[11] A. Henne, A. Toepfer, H.J. Luedde, R.M. Dreizler, J. Phys. B 19, L 361 (1986).

[12] J.R. Allan, J. Phys. B 19, L 683 (1986).

[13] W. Fritsch, C.D. Lin, Phys. Lett. A 123, 128 (1987).

[14] L.F. Errea, J.M. Gomez-Llorente, L. Mendez, A. Riera, J. Phys. B 20, 6089 (1987).

[15] T.G. Winter, Phys. Rev. B 21, L 19 (1988).

[16] M. Kimura, J. Phys. B 21, L 19 (1988).

[17] B. Peart, K. Rinn, K.T. Dolder, J. Phys. B 16, 1461 (1983).

[18] B. Peart, K. Rinn, K.T. Dolder, J. Phys. B 16, 2381 (1983).

[19] K. Rinn, F. Melchert, E. Salzborn, J. Phys. B 18, 3783 (1985).

[20] M.F. Watts, K.F. Dunn, H.B. Gilbody, J. Phys. B 19, L 355 (1986).

[21] F. Melchert, K. Rinn, E. Salzborn, N. Grün, J. Phys. B 20, L 223 (1987).

[22] T.G. Winter, Phys. Rev. A 49, 1767 (1994).

[23] S. Bhattacharyya, K. Pathak, A. Becker, F.H.M. Faisal, Acta Phys. Pol. A 90, 1143 (1997).

[24] A. Pfeiffer, S. Krüdener, F. Melchert, L.P. Presnyakov, R. Schulze, S. Meuser, T. Bartsch, C. Brandau, K.v. Diemar, K. Huber, E. Salzborn, Nucl. Instrum. Methods Phys. Res. B 98, 240 (1995).

[25] B. Joulakian, C.D. Cappello, Phys. Rev. A 47, 3783 (1993).

[26] A. Nordsieck, Phys. Rev. 93, 785 (1954). 